\title{
Short communication: Associations of udder edema with health, milk yield, and reproduction in dairy cows in early lactation
}

\author{
E. I. Morrison, ${ }^{*}$ T. J. DeVries, $†$ and S. J. LeBlanc ${ }^{* 1}$ \\ *Department of Population Medicine, and \\ †Department of Animal Biosciences, University of Guelph, Ontario, N1G 2W1 Canada
}

\begin{abstract}
Udder edema (UE) is a common condition of cows around calving, but its effects are not well characterized. The objectives of this study were to determine the associations of UE with the incidence of health disorders and with milk yield and reproduction in dairy cows in early lactation. On 3 commercial farms, UE was scored weekly on 1,346 cows, on a scale of 0 to 3 , from $1 \mathrm{wk}$ before calving to 3 wk after calving. Among cows with complete UE scores, $30 \%$ never had edema, $12 \%$ had edema only prepartum, $11 \%$ had it only postpartum, and $48 \%$ had edema prepartum and in at least $1 \mathrm{wk}$ postpartum. Udder edema was associated with a greater incidence of clinical mastitis before $30 \mathrm{~d}$ in milk (5 vs. $2 \%$ ). Subclinical ketosis (blood $\beta$-hydroxybutyrate $\geq 1.2 \mathrm{mmol} / \mathrm{L}$ ) was more prevalent at wk 2 (11 vs. $6 \%$ ) postpartum among cows with UE. No association was observed of UE with other diseases or culling in early lactation. In a subset of 912 cows with complete UE and 3 test-days of milk yield data, differences were observed in yield at test d 1 among UE categories. Cows with UE only prepartum produced less milk (39.9 $\mathrm{kg} / \mathrm{d})$ than cows with UE postpartum only $(42.4 \mathrm{~kg} / \mathrm{d}$ ) and cows with UE both prepartum and postpartum $(41.6 \mathrm{~kg} / \mathrm{d})$, none of which differed from cows without UE $(40.9 \mathrm{~kg} / \mathrm{d})$. Udder edema was not associated with the prevalence of anovulation, or the time to or probability of pregnancy at first insemination, yet to $300 \mathrm{~d}$ in milk, cows that had UE postpartum had a shorter time from calving to pregnancy than cows without UE. The associations of UE with health and productivity are mixed, and the mechanisms underlying UE and its effects merit further investigation.
\end{abstract}

Key words: udder edema, health, transition

Received February 4, 2018.

Accepted June 25, 2018.

${ }^{1}$ Corresponding author: sleblanc@uoguelph.ca

\section{Short Communication}

Udder edema (UE) is a common yet little-investigated metabolic disorder (Melendez et al., 2006; Kojouri et al., 2015). Udder edema is the accumulation of lymphatic fluid in the interstitial space of the mammary gland and surrounding tissues (Tucker et al., 1992; Kojouri et al., 2015). The causes of UE are not clear, but it can occur when circulating lipid and lipoprotein concentrations decrease due to impairment in liver function with low DMI (Kojouri et al., 2015). Kojouri et al. (2015) found that serum concentrations of total proteins, triglycerides, cholesterol, and lipoproteins were lower in cows with UE. Other problems related to UE include difficulty with milking machine attachment, risk of teat and udder injuries, mastitis, and reduction in milk production (Melendez et al., 2006; Bacic et al., 2007).

Cows, particularly entering the first lactation, generally have some UE in late pregnancy and at parturition, but extensive edema has been shown to affect milk production and health of the udder (Malven et al., 1983). Cows with longer gestation length showed increased severity of edema (Malven et al., 1983). Malven et al. (1983) also reported an association of higher plasma concentrations of estradiol- $17 \alpha$ and estrone with increased severity of edema. Melendez et al. (2006) reported that milk yield at the first DHIA test day was $3.6 \mathrm{~kg}$ lower in cows with UE. Van Dorp et al. (1998) identified a positive genetic correlation between milk yield and UE.

The objective of this study was to measure the association of UE with the incidence of health disorders in the transition period, milk yield in early lactation, and reproductive performance. We hypothesized that UE would be associated with greater incidence of health disorders and lesser milk yield in early lactation.

The data for this observational study were collected over 1 yr on 3 commercial freestall dairy farms in Ontario with 195 to 450 milking cows, concurrent with a randomized controlled trial of a dietary supplement 
of B vitamins, reported elsewhere (Morrison, 2017). All farms used automated activity monitors for estrus detection as the primary means of reproductive management, supplemented by synchronization for timed AI. The treatment in the controlled trial had no effect on the prevalence, severity, or duration of UE, and did not modify the associations of UE with the outcomes reported here. The exposure of interest was the presence of UE (defined below as a score $\geq 2$ ). Herds were purposively selected based on milking more than 150 Holstein cows, being enrolled in DHIA milk recording, and maintaining accurate disease records. Each farm was visited weekly from November 2015 to December 2016. The University of Guelph Animal Care Committee reviewed and approved the study protocols that were accepted and followed by the herds enrolled.

Udder edema scores were assigned weekly at $1 \mathrm{wk}$ before expected calving and in each of the first $3 \mathrm{wk}$ after calving. The cows were assigned a score of 0 to 3 using a scheme developed for this study (Figure 1). Scores were assigned based on visual assessment and palpation of the udder. The scoring system was developed from those used by Dentine and McDaniel (1983), Nestor et al. (1988), and Tucker et al. (1992). Those ranged from 5- to 10-point scoring systems, which makes discrimination between scores difficult to assess properly. The scoring system developed for this study was a 4-point scale from 0 for no edema to 3 for severe edema. Interrater agreement was assessed with 3 independent scorers at the time of the scoring development. Cohen's $\kappa$ coefficient was calculated and substantial agreement of $\kappa=0.76$ was found between raters 1 and 2 , and raters 2 and 3, with almost perfect agreement of $\kappa=0.88$ between raters 1 and 3 .

Blood samples were collected from the coccygeal vessels into evacuated tubes without anticoagulant (Vacutainer, Becton Dickinson and Company, Franklin Lakes, NJ) between 4 and $10 \mathrm{~d}$ before the expected calving date and between 1 and 7 DIM to measure serum nonesterified fatty acids (NEFA). Serum NEFA was measured with a Cobas 6000 c501 (Roche, Basel, Switzerland) biochemistry analyzer using the Randox NEFA kit at the Animal Health Laboratory, University of Guelph. Blood samples were taken once each week for the first 3 wk postpartum to measure blood BHB with a validated point-of-care meter (Precision Xtra, Abbott Laboratories, Mississauga, Ontario, Canada). Blood samples to measure serum progesterone were collected at 6 and 8 wk postpartum from the coccygeal vessels. Progesterone was measured by the investigators using a validated (Broes and LeBlanc, 2014) ELISA kit (Ovucheck Plasma, Biovet, St. Hyacinthe, Quebec, Canada). Cows with serum progesterone $<1 \mathrm{ng} /$ $\mathrm{mL}$ in both samples were classified as anovular. Body condition was scored on a 5-point scale (Edmonson et al., 1989) 3 wk before and 3 wk after calving. Cows were examined at wk 5 postpartum for purulent vaginal discharge using a Metricheck device (Simcrotech, Hamilton, New Zealand). Cows with muco-purulent or purulent discharge were classified as having purulent vaginal discharge. Cows sold for dairy, domestic, or export purposes were not counted as culls within the first 30 DIM.

A total of 1,346 cows were enrolled in the study. The sample size was based on the underlying randomized trial. Data on disease occurrences, culling, and reproductive performance were extracted from each farm's computerized records (DairyComp 305, Valley Ag Software, Tulare, CA). Data for milk yield $(\mathrm{kg} / \mathrm{d})$, milk fat (\%), milk protein (\%), and SCC were recorded from DHIA records for the first 3 test-day samples (CanWest DHI, Guelph, ON, Canada).

All statistical analyses were completed in SAS (version 9.4, SAS Institute Inc., Cary, NC). Linear regression models (MIXED procedure in SAS) were used to evaluate all continuous outcomes (milk production data; BHB and NEFA concentrations) for associations with UE. Where relevant for blood BHB concentrations and milk yield, repeated measures were accounted for with an autoregressive type 1 covariance structure, selected based on providing the lowest Akaike's information criterion for the final model. In the milk yield models, covariates (DIM, parity, milk protein \%, milkfat \%, and SCC) were controlled for and removed from the model if they were not significant $(P>0.05)$. Residuals for the models were graphically examined and variables with nonnormal distributions had the outcome log-transformed for analysis. For categorical outcomes (clinical disease, ketosis, culling $<30$ DIM, and pregnancy at first AI), UE as an independent variable was evaluated using logistic regression models (MIXED or GLIMMIX procedure in SAS). Univariable analysis of the association of UE with categorical outcomes was done with chi-squared statistics before building models. Categorical outcomes from continuous data [BHB $\geq 1.2 \mathrm{mmol} / \mathrm{L}, \mathrm{NEFA} \geq 0.4$ (the week before calving) and $\geq 0.7$ or $\geq 1.0 \mathrm{mmol} / \mathrm{L}$ (in wk 1 after calving), and ovulation status] were evaluated using logistic regression models (GLIMMIX procedure in SAS).

Preliminary screening was done to determine the appropriate cut point of UE score to classify UE. Cut points at scores 1 and 2 were compared, and based on associations with the outcomes listed, a cut point of 2 was selected.

Each model was initially run with treatment (from the underlying clinical trial), parity (first, second, or third or greater), farm, and all possible interactions with UE status as fixed effects and then was reduced 


\begin{tabular}{l} 
Score Definition \\
\hline 0 \\
None - No edema present \\
Slight - Edema beginning to reduce the appearance of the \\
medial suspensory ligament; uneven quarters \\
Moderate - Edema obstructing the appearance of the \\
medial suspensory ligament, beginning to extend toward \\
the front of the udder and beginning to accumulate near \\
the navel
\end{tabular}

Figure 1. Udder edema scoring system developed for a study investigating the associations of udder edema with health, milk yield, and reproduction in dairy cows. Color version available online. 
if the variables were not significant $(P>0.05)$. In each model, each weekly edema classification was offered to the model separately and then significant scores were placed in the model together and the model was reduced if variables were not significant. A summary predictor variable for UE score $\geq 2$ on at least one occasion, and a categorical variable with 4 classes of UE (described below) were also examined. Treatment (placebo or B vitamin supplementation) was not associated with UE or any of the outcomes, but was forced into all models because of the underlying trial.

Time-to-event outcomes (intervals from calving to first AI, to pregnancy, and to culling $<30$ DIM) were evaluated using Kaplan-Meier survival analysis (LIFETEST procedure in SAS). Multivariable models employed Cox proportional hazard regression analysis (PHREG procedure in SAS).

Sixty-six percent of cows had UE (score $\geq 2$ ) at least once, including $6 \%$ that had UE at all $4 \mathrm{wk}, 14 \%$ in 3 of the weeks, $20 \%$ in 2 , and $26 \%$ on only 1 occasion. Among cows with UE in the week before calving, $65 \%$ had UE in wk 1 postcalving, $70 \%$ in wk 2, and $79 \%$ had edema in wk 3. An association of parity with UE $(P<0.001)$ was observed; $86 \%$ of first-parity cows had UE, whereas second-parity (56\%) and third-parity cows $(59 \%)$ had lower prevalence of edema (Table 1). Farm had no association with the prevalence of UE.

Associations of UE with ketosis and blood NEFA concentrations were present. There was no association of having UE with blood BHB in wk 1 postpartum, but cows with UE had higher concentrations (mean $\pm \mathrm{SE}$ ) of BHB in blood at wk $2(0.14 \pm 0.01$ vs. $0.08 \pm 0.01$ $\mathrm{mmol} / \mathrm{L} ; P=0.002)$ and wk $3(0.09 \pm 0.01$ vs. 0.06 $\pm 0.02 \mathrm{mmol} / \mathrm{L} ; P=0.04)$. The prevalence of ketosis (blood $\mathrm{BHB} \geq 1.2 \mathrm{mmol} / \mathrm{L})$ at wk 2 was greater $(P=$ 0.01 ) among cows that experienced UE (11 vs. $6 \%$ ). Udder edema at wk -1 was associated with higher serum concentration of NEFA $(0.34 \pm 0.02$ vs. $0.17 \pm 0.02$ $\mathrm{mmol} / \mathrm{L} ; P<0.001)$ and with a higher prevalence of elevated NEFA $(\geq 0.4 \mathrm{mmol} / \mathrm{L} ; 33$ vs. $12 \%, P<0.001)$ at the same time. Prepartum UE was also associated with higher serum concentration of NEFA in the first week postpartum $(0.87 \pm 0.2$ vs. $0.79 \pm 0.2 \mathrm{mmol} / \mathrm{L}$, $P=0.007)$, but not with the prevalence of cows with elevated NEFA $(>0.7 \mathrm{mmol} / \mathrm{L} ; 54 \%, P=0.67)$ at wk 1 . No association $(P=0.33)$ was observed of UE at wk 1 with serum NEFA concentration at the same time $(0.79$ $\pm 0.02 \mathrm{mmol} / \mathrm{L})$.

Udder edema was associated with a higher incidence risk of clinical mastitis in early lactation (Table 2). The median occurrence of first cases of mastitis was 8 DIM (range 1 to $26 \mathrm{DIM}$ ), and no weeks or classes of UE were specifically associated with mastitis (data not shown). No other associations of UE with clinical disease or culling in early lactation were observed.

Because DHIA milk tests largely occurred after assessment of UE (the first test day was at $22 \pm 11$ DIM), we assessed the association of UE with milk production in the subset of 912 cows that had complete $(\mathrm{n}=4) \mathrm{UE}$ scores and DHIA ( $\mathrm{n}=3$ tests) data. For this analysis, UE was classified as none, prepartum only, postpartum only, or both. There tended $(P=0.09)$ to be an interaction of UE class with DHIA test day. Therefore the analyses were stratified by test day, and (accounting for covariates) significant differences among UE classes were found at DHIA test d 1 only (Table 3 ), such that cows with UE only prepartum produced less milk than cows with UE postpartum or both pre- and postpartum.

Reproduction outcomes were also assessed using the 4 categories of UE. No difference $(P>0.18)$ was observed in the prevalence (LSM $\pm \mathrm{SE}$ ) of ovulation among cows with no UE ( $82 \pm 3 \%)$, UE prepartum only $(85 \pm 3 \%)$, UE postpartum only $(81 \pm 4 \%)$, or both pre- and postpartum $(80 \pm 4 \%)$. Similarly, no differences (Wilcoxon $P=0.80)$ were observed among UE categories in the median time to first AI (61 to $63 \pm 5 \mathrm{~d}$ ). Accounting for parity and farm, no difference $(P>0.15)$ was observed in the proportion of cows pregnant at first AI among cows with no UE $(32 \pm 4 \%)$, UE prepartum only ( $42 \pm$ $6 \%$ ), UE postpartum only (36 $\pm 7 \%)$, or both pre- and postpartum $(37 \pm 3 \%)$. However, accounting for farm, treatment, and parity, over 300 DIM, time from calving to pregnancy was shorter in cows with UE postpartum (hazard ratio of $1.5,95 \% \mathrm{CI}, 1.2$ to $2.1 ; P=0.004$ ) or

Table 1. Prevalence of udder edema (UE; $\%$ of herd with UE $\geq 2$ ) by parity in 3 commercial dairy herds

\begin{tabular}{lccccc}
\hline & & \multicolumn{4}{c}{$\begin{array}{l}\text { Week relative to calving } \\
\text { in which UE was scored }\end{array}$} \\
\cline { 3 - 6 } Item & $\begin{array}{c}\text { Total } \\
\text { scores (no.) }\end{array}$ & -1 & 1 & 2 & 3 \\
\hline Parity & & & & & \\
1 & 1,890 & 84.0 & 67.3 & 39.3 & 18.3 \\
2 & 1,040 & 21.2 & 46.6 & 24.2 & 11.3 \\
3 & 1,725 & 35.2 & 46.4 & 25.2 & 15.7 \\
$P$-value & & $<0.001$ & $<0.001$ & $<0.001$ & 0.04 \\
\hline
\end{tabular}


Table 2. The association of udder edema ( core $\geq 2$ ) assessed at wk $-1,1,2$, and 3 relative to calving with the incidence of clinical disease or culling in 3 commercial dairy herds

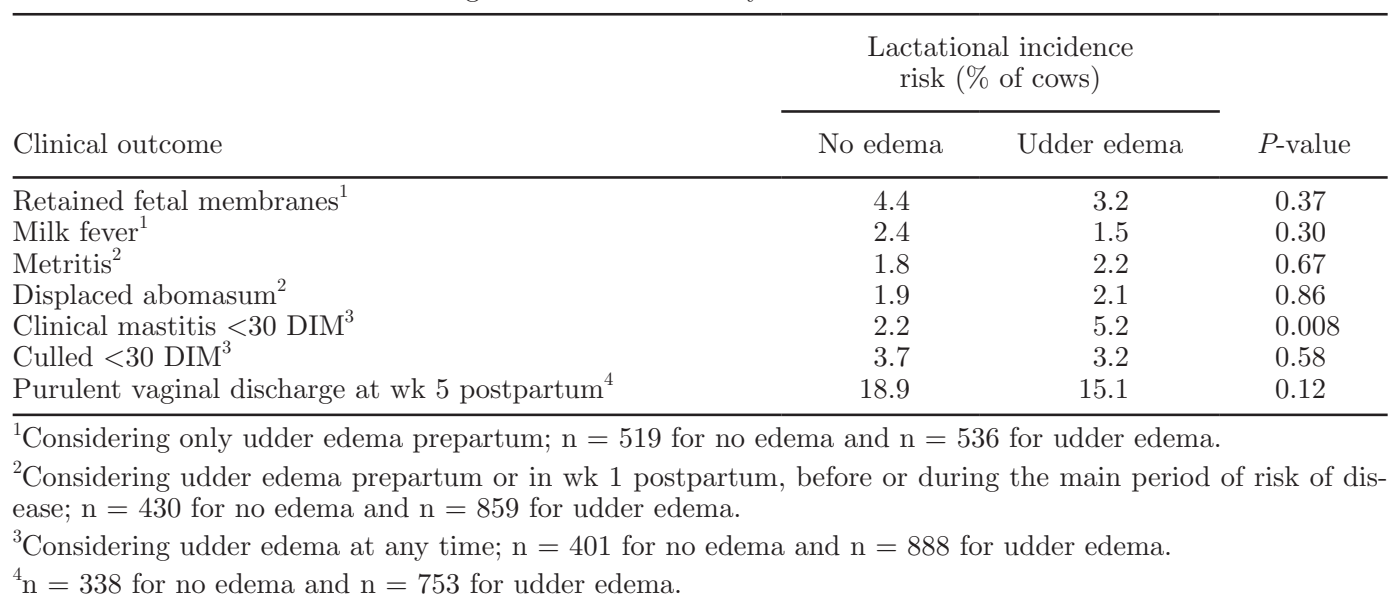

with UE both pre- and postpartum (hazard ratio of 1.4, 95\% CI, 1.2 to $1.8 ; P=0.0004)$ compared with those without UE. Time to pregnancy in cows with UE only before calving did not differ from those without UE.

We found that UE was very common, particularly, as expected, in primiparous cows, similar to Dentine and McDaniel (1983) and Kojouri et al. (2015). The novel UE scoring scheme developed here was robust (high agreement among raters) and easy to implement. Despite having a large sample, data were insufficient relative to the outcomes to detect differences in associations with the study outcomes among the many combinations of patterns of the timing of the onset, duration, and severity of UE.

Melendez et al. (2006) found that UE was not associated with displaced abomasum, metritis, or mastitis. In the present study, we found no association of UE with retained fetal membranes, milk fever, displaced abomasum, metritis, purulent vaginal discharge, or early culling. We observed conditional associations of UE with modestly higher prevalence of ketosis. Specifically, cows that had UE on at least one occasion were more likely to have ketosis at wk 2 postpartum. The pathophysiology of UE is not well described, but it is unclear how it would relate directly to that of ketosis. We speculate that UE might cause some physical discomfort, which might lead to decreased DMI and in turn contribute to the risk of ketosis. The association of UE with higher odds of clinical mastitis in early lactation was consistent with our hypothesis and is likely explained in part by poorer milk-out, worse teat end condition, and impaired teat sphincter function in cows with UE, all of which would plausibly increase the risk of IMI.

Others (Dentine and McDaniel, 1983; Melendez et al., 2006; Kojouri et al., 2015) have shown that cows with UE produced less milk at the first DHIA test day. We also found effects of UE at the first DHIA test day only, such that cows with edema only before calving made 1.5 to $2.5 \mathrm{~kg} / \mathrm{d}$ less component-adjusted milk than cows with UE postpartum only or both pre- and postpartum. A reduction in milk yield would presumably be attributable in part to inhibition of milk secretion from increased pressure in the udder. Conversely, a positive genetic correlation between UE and milk yield has been reported (Van Dorp et al., 1998). We also found that cows with UE were more likely to have clinical mastitis before 30 DIM, which may in turn reduce milk yield. Because clinical mastitis was an intervening variable

Table 3. The association of udder edema (score $\geq 2$ ) assessed weekly from $1 \mathrm{wk}$ before to $3 \mathrm{wk}$ after calving with milk yield (LSM $\pm \mathrm{SE}$ ) at the first DHIA test of lactation in 912 cows in 3 commercial dairy herds ${ }^{1}$

\begin{tabular}{lrc}
\hline Udder edema status & No. $(\%)$ & $\begin{array}{c}\text { Milk yield }^{2} \\
(\mathrm{~kg} / \mathrm{d})\end{array}$ \\
\hline Unaffected & $271(29.7)$ & $40.9 \pm 0.5$ \\
Edema prepartum only & $109(12.0)$ & $39.9 \pm 0.8^{\mathrm{a}, \mathrm{x}}$ \\
Edema postpartum only (on at least 1 occasion) & $99(10.9)$ & $42.4 \pm 0.8^{\mathrm{b}}$ \\
Edema prepartum and on at least 1 occasion postpartum & $433(47.5)$ & $41.6 \pm 0.4^{\mathrm{y}}$ \\
\hline${ }^{1}$ The model accounts for fixed effects of farm, parity, DIM at test day, milk fat and protein percentages, and \\
SCC. ${ }^{2}$ Pairwise differences: ${ }^{\mathrm{a}, \mathrm{b}} \mathrm{P}=0.02 ;{ }^{\mathrm{x}, \mathrm{y}} \mathrm{P}=0.04$.
\end{tabular}


between UE and test day milk yield, we cannot accurately partition the effects of UE and mastitis. Further research on the mechanisms underlying UE and how UE relates to milk yield is warranted.

We hypothesized that if UE were associated with clinical disease, consequent associations might be present with reproduction before and at first insemination. The overall incidence of the conditions with which UE was associated with greater risk (mastitis, and ketosis in wk 2 postpartum) was low, and so unlikely to translate to substantial effects on pregnancy at first AI. Despite the lack of association of UE with the prevalence of ovulation by wk 8 postpartum or with pregnancy at first AI, over 300 DIM an association of postpartum UE with a shorter median time to pregnancy was observed. Again, a mechanism to explain this association is elusive and this relationship should be confirmed in future studies.

In summary, we established a new, simple, and reliable scheme for scoring UE, which could easily be added to data collection in other large-scale studies of health in transition cows. Udder edema was associated with increased incidence of clinical mastitis in early lactation and with a modest increase in the prevalence of ketosis at wk 2 postpartum, but not with other diseases or culling. Edema that was present the week before calving was associated with a higher prevalence of concurrently elevated serum NEFA concentration. These mixed associations merit confirmation and investigation of the underlying mechanisms of UE and its potential effects on DMI, mastitis, ketosis, and milk yield.

\section{ACKNOWLEDGMENTS}

This work was funded as part of a controlled trial supported by Jefo Nutrition Inc. (St. Hyacinthe, Quebec, Canada).

\section{REFERENCES}

Bacic, G., T. Karadjole, N. Macesic, and M. Karadjole. 2007. A brief review of etiology and nutritional prevention of metabolic disorders in dairy cattle. Vet. Arh. 77:567-577.

Broes, A., and S. J. LeBlanc. 2014. Comparison of commercial progesterone assays for evaluation of luteal status in dairy cows. Can. Vet. J. 55:582-584.

Dentine, M. R., and B. T. McDaniel. 1983. Variation of edema scores from herd-year, age, calving month and sire. J. Dairy Sci. 66:23912399 .

Edmonson, A. J., I. J. Lean, L. D. Weaver, T. Farver, and G. Webster. 1989. A body condition scoring chart for Holstein dairy cows. J. Dairy Sci. 72:68-78.

Kojouri, G. A., M. M. Pouryeganeh, S. Nekouei, and S. Nazifi. 2015. Udder edema and association with some serum biochemical measures and dietary factors in first calving cows. Iran J. Vet. Res. 16:345-349.

Malven, P. V., R. E. Erb, M. F. D'amico, T. S. Stewart, and B. P. Chew. 1983. Factors associated with edema of the mammary gland in primigravid dairy heifers. J. Dairy Sci. 66:246-252.

Melendez, P., C. C. Hofer, and G. A. Donovan. 2006. Risk factors for udder edema and its association with lactation performance on primiparous Holstein cows in a large Florida herd, USA. Prev. Vet. Med. 76:211-221.

Morrison, E. I. 2017. Effect of B vitamin supplementation on health, production and reproduction in transition dairy cows. MSc Thesis. Population Medicine, University of Guelph, Guelph, Ontario, Canada.

Nester, K. E., R. W. Hemken, and R. J. Harmon. 1988. Influence of sodium chloride and potassium bicarbonate on udder edema and selected blood parameters. J. Dairy Sci. 71:366-372.

Tucker, W. B., G. D. Adams, M. Lema, M. Aslam, S. Shin, P. Le Ruyet, and D. L. Weeks. 1992. Evaluation of a system for rating edema in dairy cattle. J. Dairy Sci. 75:2382-2387.

Van Dorp, T. E., J. C. M. Dekkers, S. W. Martin, and J. P. T. M. Noordhuizen. 1998. Genetic parameters of health disorders, and relationships with 305-day milk yield and conformation traits of registered Holstein cows. J. Dairy Sci. 81:2264-2270. 